\title{
A Proposal on Wireless Ad Hoc Network Controlled Rovers
}

\author{
Jay Rungta \\ Student \\ Mukesh Patel School of \\ Technology Management and \\ Engineering, \\ Mumbai
}

\author{
Manali Satghar \\ Student \\ Mukesh Patel School of \\ Technology Management and \\ Engineering, \\ Mumbai
}

\author{
Anish Prabhu \\ Student \\ Mukesh Patel School of \\ Technology Management and \\ Engineering, \\ Mumbai
}

\author{
Vineetkumar Sanu \\ Student \\ Mukesh Patel School of Technology Management \\ and Engineering, \\ Mumbai
}

\author{
Supriya Agrawal \\ Faculty Mentor \\ Mukesh Patel School of Technology Management \\ and Engineering, \\ Mumbai
}

\begin{abstract}
An Ad Hoc network is a network which does not rely on any preexisting infrastructure. It is a network that is created to serve only a particular purpose. Wireless Ad Hoc networks consist of nodes that are interconnected and transmit data wirelessly. This paper dis- cusses about an application of Wireless Ad Hoc Networks to build a system of Rovers. The primary use of these Rovers shall be to pro- vide a means to survey areas where human intervention may not be possible at the time. Such areas include disaster struck buildings, enemy occupied buildings, possible radioactive areas, etc. This paper reviews several techniques that may help in the construction of such a system. Their pros and cons are discussed and a possible solution to make an optimal system of Rovers is proposed.
\end{abstract}

\section{General Terms}

3G Shield, Ad Hoc Networks, Arduino, Rovers, Wireless Ad Hoc Networks

\section{Keywords}

3G Shield, Ad Hoc Networks, Arduino, Energy Saving, GSM, MANET, Rover, VANET, WANET, Wi-Fi

\section{INTRODUCTION}

Wireless Ad Hoc networks (WANETs) are formed dynamically by an autonomous system of mobile nodes that are connected via wireless links. Wireless Ad Hoc networks can be used in n number of ways. Some of their applications are as MANETs (Mobile Ad Hoc Networks) and VANETs (Vehicular Ad Hoc Networks). The application of the system depends on the nature of the nodes. The focus of this paper is when the nodes are Rovers. The uses of such a system are endless. They can be used to access disaster struck areas for search and rescue purposes; they have military applications for search and reconnaissance; security applications for surveillance, and several more. All of the above applications require a system of mobile nodes that can be controlled by a central entity.

Although such a system is easily accomplishable through a Mobile Ad Hoc Network (MANET), several challenges need to be tackled. The design process of an individual Rover itself is a huge challenge. This includes coupling of various components of the Rover in a manner that increases performance and saves energy at the same time, ensuring that the Rover is robust enough to bear all sorts of environmental hazards its applications may have, programming the microcontroller of the Rover, and finally testing it under various circumstances. The second major challenge is designing the architecture of the Wireless Ad Hoc Network that will control the Rovers. The fundamental requirements of the system are that the Rovers must have a strong network connection at all times, since they shall be used in critical situations such as disaster mitigation, military zones, radioactive areas, etc. The power consumed by the system should be as minimum as possible, to increase the survivability of each node in the system. This is desirable as the applications of this system usually last for long hours. Moreover, the Rovers need to assemble back to a point where extraction would be possible. The design of such an Ad Hoc Network is the main focus of this paper.

Section 2 discusses pre-existing research papers that have implemented Wireless Ad Hoc Networks in different manners. Each technique discussed provides different methods that can be employed to obtain an optimal system. Section 3 describes the proposed architecture and components of the Rover in the proposed system.

\section{EXISTING TECHNIQUES}

\subsection{Wi-Fi Enabled Smart Robot}

In the first architecture proposed in the paper Wi-Fi Enabled Smart Robot [1], we can maneuver and control the Rover via a webpage. To make this viable, an Arduino micro-controller board with an Arduino Ethernet Shield is used to control and navigate the Rover to the desired path. The Rover can be controlled via a joystick which has front, left, right and back functions. In this project, the authors have designed an interface to the switch control mode which suits their requirements. Finally they successfully interfaced the Rover with the Arduino and controlled it in the wireless environment with the help of the control functions on the joystick through a webpage. 


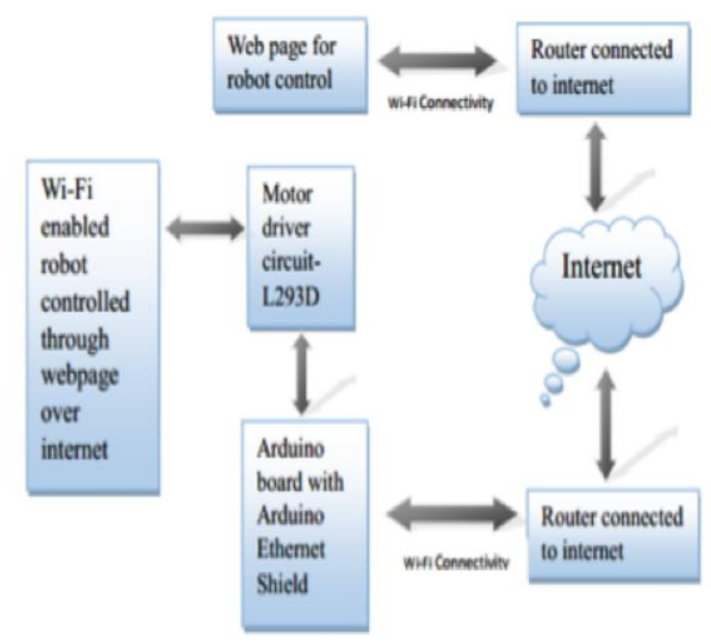

Figure 1: Functional Block Diagram of the Wi-Fi Model [1]

Fig. 1 shows the block diagram of the architecture. The robot can be controlled and navigated via a custom designed webpage as clearly evident from the diagram. When the URL of the website is entered in the web browser, the web page containing the various controls of Rover gets loaded. Upon clicking on the respective direction control buttons, the control packets are created. These packets are transferred via the Internet and are received by the Arduino Ethernet Shield serially via the router. The data is further transferred from the Ethernet Shield through an SPI interface into the Arduino board and the designated directions are worked up using the motor shield to navigate the robot.

The advantage of this architecture is that the Rover can be controlled using any hand-held device that has an Internet connection. However, the drawback is that the architecture relies heavily on Wi-Fi routers, which often have a limited range of maneuverability of the Rover and also the power consumption is high as live streaming video from the Rover's cameras may drain a significant amount of energy from the batteries.

\subsection{GSM Network Model}

The second architecture proposed in the paper Implementation of Unmanned Vehicle using GSM Network with Arduino [2], cellular phones using GSM network are used to control an unmanned vehicle. This technique overcomes all the shortcomings of the aforementioned Wi-Fi Model like network coverage issue and communication problems. This technique is solely dependent on worldwide GSM network, which is up and running $24 \times 7$ and has very minimal interference. The unmanned vehicle is interfaced with a GSM network consisting of a GSM enabled cellular phone, DTMF decoder, Arduino Uno microcontroller board and two motors. Any GSM enabled cellular phone can be used, including low end black and white feature phones with basic function to high end Android OS / iOS operated smartphones. No special requirements are needed for two cellular phones. One is used as a transmitter (handheld remote controlled device) and other is used as a receiver interfaced with the unmanned vehicle.

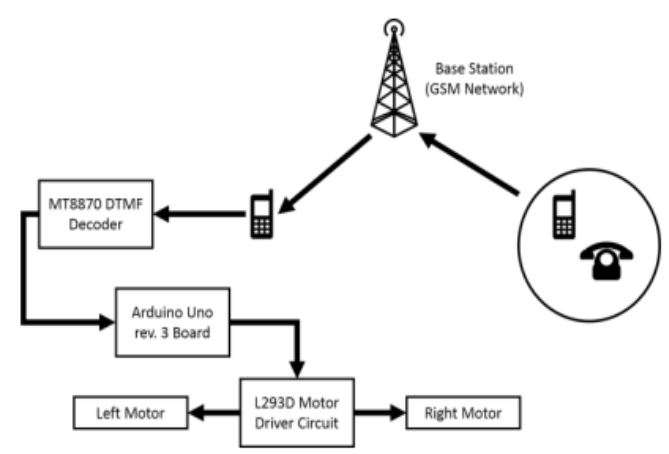

Figure 2: Functional Block Diagram of the GSM Model [2]

Fig. 2 depicts the overall functionality of the system. A call is placed either from any kind of phone to the cellular phone present in the Rover. The call is placed via the GSM network. Upon answering the call on the receiving cell phone, the connection between the sender and Rover is established successfully. During the call, if any button is pressed on the keypad of the dialing phone, a tone which corresponds to the number key pressed is generated. This tone is called as DTMF (Dual Tone Multiple Frequency) and the generated code of the key button pressed is sent to the DTMF decoder through the cellular phone's $3.5 \mathrm{~mm}$ audio jack headphone output. The DTMF microcontroller then decodes the tones to give binary digits which are in turn sent to the Arduino Uno microcontroller board. The Arduino Uno board then makes decision based on the input from the DTMF decoder and outputs the signals to the attached motors. The two motors help the Rover to maneuver forwards, backwards, left and right.

This architecture solves the problem of the limited range by using GSM network. It can be used in civil operations like monitoring and surveillance thus decreasing the cost involvement for the human workforce. However in this architecture, the GSM networks aren't available in remote areas hence reachability is hindered. Also, GSM networks use far greater power than Wi-Fi routers.

\subsection{Soldier-Robot Ad-Hoc Network}

In the paper A Soldier-Robot Ad Hoc Network [3], solider robots are controlled using a MANET (Mobile Ad-Hoc Network). It can help in supporting reconnaissance, surveillance and target acquisition (RSTA) capabilities. MANET do not depend on any infrastructure and it also possess routing capabilities to self- configure and adapt different network topologies and the ad-hoc network itself uses multi-hop communication between nodes also by MANET the communication can be further improvised. In this Linksys wireless LAN routers (models WRT54GS and WRTSL54GS) to develop ad-hoc nodes. The Linksys routers are built with an internal $100 \mathrm{~mW}$ amplifier which allows for long distance wireless operation without the use of highpower antennas and the Integrated MANET capabilities onto two robotic platforms, the iRobot PackBot Explorer and iRobot/John Deere R- Gator. The PackBot Explorer is a small unmanned ground vehicle (SUGV) which is equipped with video cameras for intelligence, reconnaissance, and surveillance. The R-Gator robot is a larger platform developed for transport and RSTA purposes and it is capable of being driven manually or teleoperated. Has more space hence has 
more powerful antennas. For implementing the network, they have used IEEE 802.11g network and Linksys wireless LAN routers for mobility and speed. The test was performed on the rover and they achieved a $75 \%$ to $80 \%$ maximum operational distance and it also maintained a link with five hops among the nodes.

The advantages of this architecture is that no infrastructure is required since it is feasible in areas with low density like deserts, war zones where infrastructure is not a viable option and hence not installation cost and it also provides better mobility since The node can hop to a node inside the network and it can also communicate with other node in this network. The drawback being that there is energy limitation since due to reliance on battery power, the power consumption is more and it is practically infeasible to form a network for nodes to move freely.

\subsection{Geocast Protocol for Vehicular Ad-Hoc Networks}

The research paper Design and Evaluation of two Geocast Protocols for Vehicular Ad-hoc Networks [4] discusses use of VANET (Vehicular Ad-Hoc Networks) for getting the exact location of the rover. In this, each vehicle is a node and it is more efficient than GPS but it requires road side units to give exact information. VANET is also used for increasing intervehicle coordination and it helps us to increase the connectivity between adjacent nodes. The author has used the simulation package SWANS, with the STRAW module. SWANS is a simulator for mobile ad-hoc networks in JAVA. Each vehicle is installed with a wireless router system that can communicate and transmit data between other vehicles (IVT) and also between road side units. The author's architecture enables quick transmission of data between the nodes.

The advantage of this architecture is that the Geocast protocol for VANET is designed for fast communication across a large area and the robust vehicular routing protocol provides a routing service for a future reliable transport protocol enabling Internet applications. Whereas the drawback being that there is increase in network congestion and it gives a measurably bad performances for long distance between the source and destination.

\subsection{Energy Aware Routing}

The research paper Energy Aware Routing for Low Energy Ad Hoc Sensor Networks [5] focuses on network survivability as longer as possible and by using Energy aware routing protocol, the nodes uses not only one single optimal path for communication but keeps a set of good paths and chooses one based on a probabilistic fashion. In this protocol, they try to use PicoNodes which are light weight, low cost network elements, smaller than one cubic centimeter and weight is less than $100 \mathrm{gms}$. Trying to network such large number of low powered nodes is a challenging thing. The authors also try to make the nodes self-powered through their environment, so that the nodes last longer.

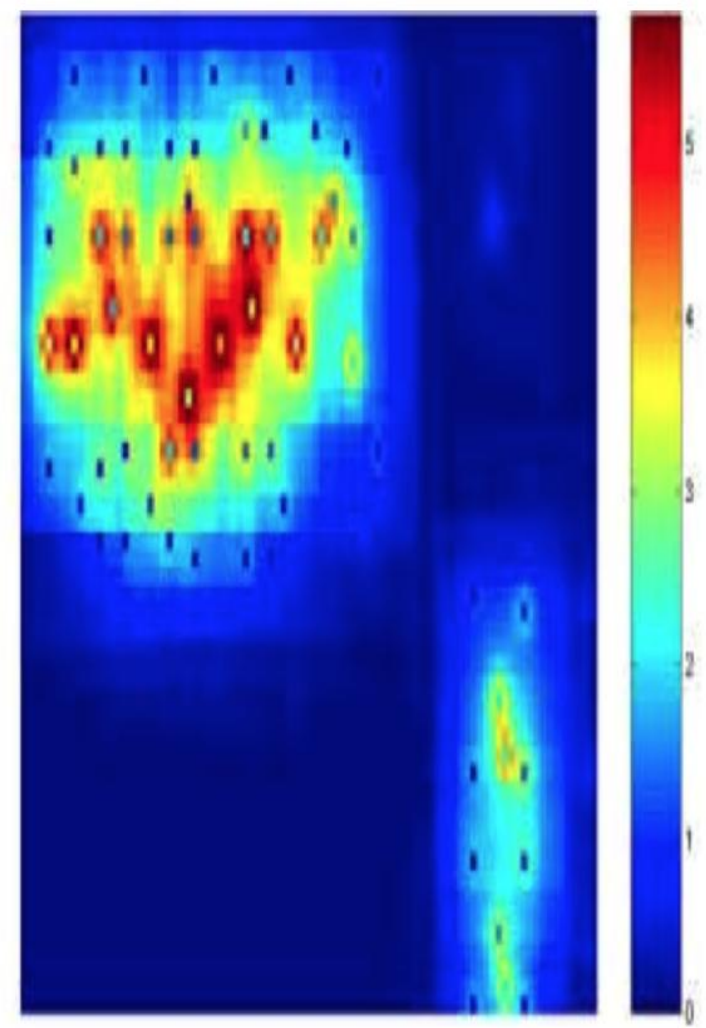

Figure 3: Energy consumption for Energy Aware Routing [5]

Simulations were carried out in Opnet to demonstrate the increased network survivability due to energy aware routing. The simulation consisted of 76 nodes in a typical office setup. There were 65 sensors and 7 controllers that were static and 4 nodes that were mobile and among the sensors, 47 were light sensors and 18 were temperature sensors. The controllers sent out requests for data to the sensors in their region of interest and the requests programmed the light sensors to send data every 10 seconds and temperature data every 30 seconds. A heat map of the power consumed was generated for the energy aware routing architecture. It showed that energy aware routing reduces the average energy consumption per node from $14.99 \mathrm{~mJ}$ to $11.76 \mathrm{~mJ}$, an improvement of $21.5 \%$.

The advantage of this architecture is that in this a new routing protocol that is suitable for low energy and low bit rate networks is used and also a simple mechanism to send traffic though different routes helps in used which utilizes the node resources more equitably. While the drawback being that using a low energy path frequently leads to energy depletion of the nodes along that path and also the network survivability is degraded by implementing the low energy protocol. 


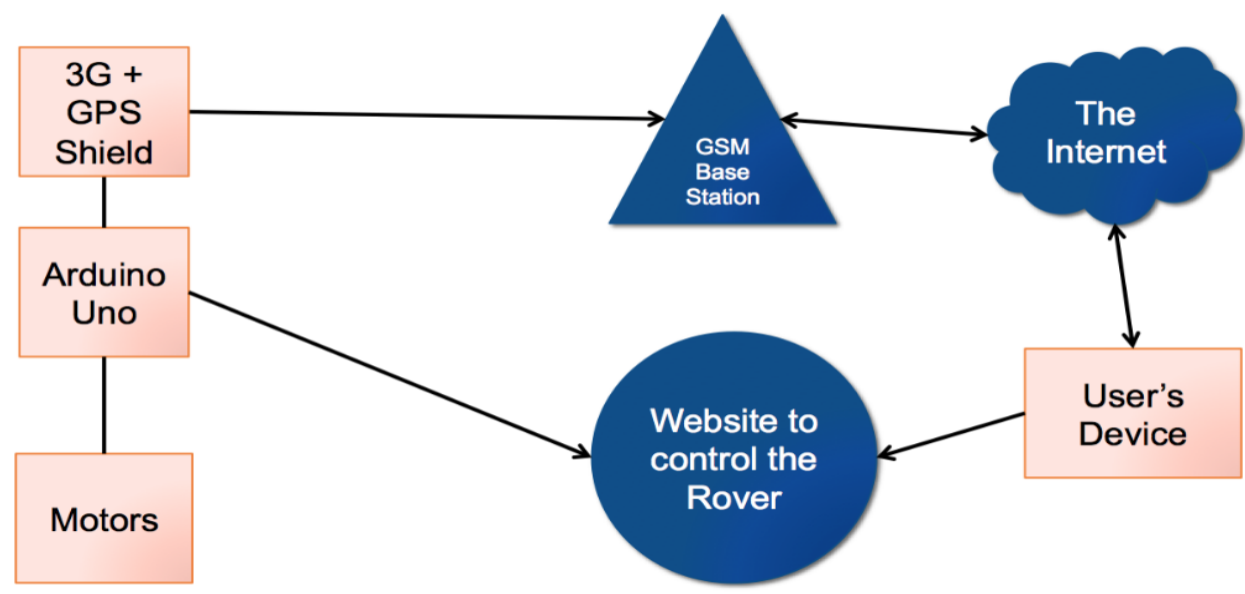

Figure 4: Proposed System Architecture

\section{PROPOSED SYSTEM}

Our proposed system will incorporate the method used in the energy aware routing technique paper [5] to minimize the energy consumption between nodes. The data transfer can be made faster by incorporating the algorithm used in the paper Geocast Protocols for VANET [4]. Further, we somehow needed to combine the powerful range of GSM networks and the low power consumption of Wi-Fi routers. The optimal solution we came up with was to use a $3 \mathrm{G}+\mathrm{GPS}$ Shield on the Rover to connect it to the Internet. $3 \mathrm{G}+\mathrm{GPS}$ Shield uses very little power and has the same range of GSM net- works. There is little research in the field of using 3G+GPS Shield with Arduino in an Ad Hoc Network, and this makes this a novel approach to tackle the prevalent problem of power consumption and range of wireless networks.

\subsection{System Architecture}

The proposed architecture can be seen in Fig. 4. It is evident that the $3 \mathrm{G}+\mathrm{GPS}$ shield is a medium to connect the Rover to the Internet. This shield uses the GSM network. Once the connection is established, it communicates with the Web Server which contains a web page using the HTTPS protocol (for security). The user controls the Rover through a web page which contains the controls. The user can connect to the web page using any Internet enabled device. This allows using phones, laptops, tablets and any other device to control the Rover. The server backend transfers the button presses to the Arduino Uno chip via the $3 \mathrm{G}+\mathrm{GPS}$ Shield. The Arduino then translates these button presses into actions to control the motors via the attached motor controller.

The backend server also contains the logic that allows selection of an optimal path to transfer the button presses data. The available paths are constantly monitored to maintain data of the energy consumption of each node.

The best path is then chosen according to the least energy consumption and fast data transmission. This ensures long survivability of the nodes and fast relay of data.

The various components of the system are explained in the following section.

\subsection{Components of the System}

Our proposed system would have the following components:

$$
\begin{array}{ll}
\text { - } & \text { Arduino Uno Chipset } \\
\text { - } & \text { 3G +GPS Shield for Arduino } \\
\text { - } & \text { Motor Controller } \\
\text { - } & \text { Rover Chassis } \\
- & \text { Motors } \\
\text { - } & \text { Web Server For Webpage } \\
- & \text { Cameras }
\end{array}
$$

The reason for using Arduino Uno as the microcontroller is speed and ease of development. The open source nature of Arduino has led to the availability of dozens of daughter cards called shields that facilitate things like Internet access, wireless networking, data logging, and device control. One can easily "plug and play" shields to the Arduino chipset. This is the main functionality that will allow us to use a data card in our Rovers. Another big advantage is that the Arduino IDE allows software development on all major platforms (Mac, PC, Linux). Moreover, Arduino provides a number of libraries to make programming the microcontroller easier.

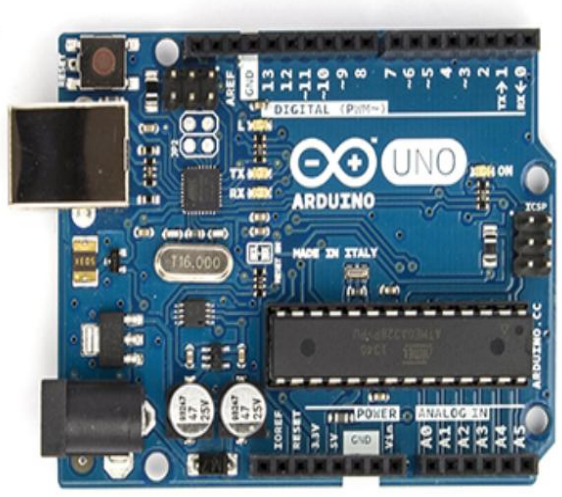

Figure 5: Front of the Arduino Uno Chipset [6] 
For implementation of the Arduino Uno chipset, we will power it via an external power supply. Leads from a battery can be inserted in the Gnd and Vin pin headers of the POWER connector. Each of the 14 digital pins on the Uno can be used as an input or output, using pinMode(), digitalWrite(), and digitalRead() functions. They operate at 5 volts. Each pin can provide or receive a maximum of $40 \mathrm{~mA}$ and has an internal pull-up resistor of 20-50 kOhms. In addition, some pins have specialized functions [6]:

Serial: 0 (RX) and 1 (TX) are used to receive (RX) and transmit (TX) TTL serial data.

External Interrupts: 2 and 3. These pins can be configured to trigger an interrupt on a low value, a rising or falling edge, or a change in value.

PWM: 3, 5, 6, 9, 10, and 11. Provide 8-bit PWM output with the analogWrite() function.

SPI: 10 (SS), 11 (MOSI), 12 (MISO), 13 (SCK). These pins support SPI communication using the SPI library.

LED: 13. There is a built-in LED connected to digital pin 13 . When the pin is HIGH value, the LED is on, when the pin is LOW, it's off.

The Arduino Uno can be programmed with the Arduino software. The ATmega328 on the Arduino Uno comes pre burned with a boot loader that allows you to upload new code to it without the use of an external hardware programmer. It communicates using the original STK500 protocol.[6]

The 3G+GPS shield for Arduino [7] is the daughter circuit that shall be used as a data card. This shield is created and manufactured by Cooking Hacks, a website for electronic DIY enthusiasts. This shield allows connection to $3 \mathrm{G}$ cellular networks, communication to webservers using HTTP and HTTPS protocols and even storage of data in microSD cards. It has all GSM antennas inbuilt and only requires a SIM card with an active $3 \mathrm{G}$ internet connection. This makes it an ideal candidate for allowing communication of the Arduino Uno with the Webpage that will be used for controlling the Rovers. The shield also allows sending video and photographs to the webserver, enabling surveillance.

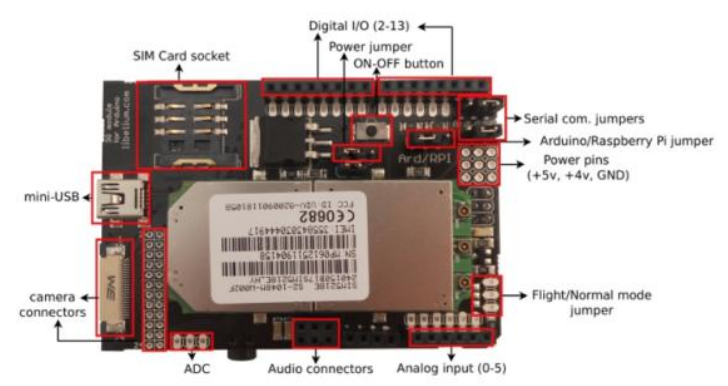

Figure 6: Components of the 3G+GPS Shield for Arduino [7]

The 3G module used is the SIM5218 from SIMCOM. The power supply of SIM5218 is from a single voltage source of $3.4 \mathrm{v}$ to $4.2 \mathrm{v}$. It supports SIM cards $3 \mathrm{~V}$ and $1.8 \mathrm{~V}$. Connecting and configuring it with the Arduino Uno chipset is fairly simple and easily accomplishable. Hence the Arduino Uno chipset and the 3G+GPS Shield for Arduino form the heart of the structure of the Rovers.

\section{CONCLUSION}

We might face several challenges ahead during the implementation phase, but our main challenge was to first identify and construct a suitable and robust architecture. The proposed architecture should be able to withstand environmental constraints and work efficiently on low power. The range of the architecture should also be maximum. The video feeds should be bug and delay free. The architecture we have come up with overcomes several of these challenges, but still needs to be tested using simulations to actually find out its effectiveness.

\section{FUTURE WORK}

We have a well-modeled and strategic plan to implement the system in various fields of applications viz. firefighting, radiation leaks and security purposes. We plan to have a separate type of Rover for each of these applications which will include gas detectors, smoke detectors, radiation detectors and a metal detector for serving the requirements in these applications. We are currently working on an optimal design of the Rover prototype for the purpose of firefighting. The next plan of action would be to construct the Rover, set up the website to control it, link the two, and finally test it. Testing of the system shall be done extensively to ensure its survivability in critical situations and hazardous environments.

\section{REFERENCES}

[1] Shashank U Moro. Wifi enabled smart robot. Science, Engineering and Technology Conference, At VIT University, Vellore, 2013.

[2] Amey Kelkar. Implementation of unmanned vehicle using gsm network with Arduino. International Journal of Advanced Research in Computer Science and Software Engineering, 2014.

[3] Brian B Luu, Barry J O'Brien, David G Baran, and Rommie L Hardy. A soldier-robot ad hoc network. In Pervasive Computing and Communications Workshops, 2007. PerCom Workshops' 07. Fifth Annual IEEE International Conference on, pages 558-563. IEEE, 2007.

[4] Maria Kihl, Mihail Sichitiu, and Harsvardhan Joshi. Design and evaluation of two geocast protocols for vehicular ad-hoc networks. Journal of Internet Engineering, 2008.

[5] Rahul C Shah and Jan M Rabaey. Energy aware routing for low energy ad hoc sensor networks. In Wireless Communications and Networking Conference, 2002. WCNC2002. 2002 IEEE, volume 1, pages 350-355. IEEE, 2002.

[6] Arduino. Arduino uno chipset. Online at http://www.arduino.cc/en/main/arduinoBoardUnon/ardu inoBoardUno.

[7] Cooking Hacks. $3 g+$ gps shield for arduino. Online at: http://www.cookinghacks.com/documentation/tutorials/arduino-3g-gprsgsm 